\title{
ANALYSIS OF NUCLEATION AND GROWTH WITH THE MODEL FOR DIFFUSION- CONTROLLED PRECIPITATION REACTIONS BASED ON THE EXTENDED VOLUME CONCEPT
}

\author{
M.J. Starink*
}

Materials Research Group, Engineering Sciences, Faculty of Engineering and the Environment, University of Southampton, Southampton S017 1BJ, UK

\footnotetext{
This version is author's version which differs from the publisher version (J. Alloys and Compounds 630, 2015, pp. 250-255 DOI: 10.1016/j.jallcom.2015.01.045) in:

- minor typographical and wording changes

- $\quad$ this version contains higher resolution figures

- $\quad$ the published version contains 3 additional sentences

- $\quad$ there are no keywords in this version
}

\begin{abstract}
Recently [M.J. Starink, Thermochim Acta 596, 2014, 109-119] a new model for diffusioncontrolled precipitation reactions based on the extended volume concept was derived. The model leads to an analytical equation describing the relation between the fraction transformed, $\alpha$, the reaction time, $t$, and the reaction exponent, $n$, as:$$
\alpha=\left\{\exp \left(-2\left(k_{1} t\right)^{n}\right)-1\right\} /\left(2\left(k_{1} t\right)^{n}\right)+1
$$

In the present work, new analysis methods are derived which allow determination of the reaction exponent $n$. The new methods are applied to analysis of nucleation and it is shown that generally during a reaction with growth in 3 dimensions there are only 2 modes: either the nucleation rate in the extended volume is constant or it is negligibly small. A new approach to the interaction of diffusion-controlled growth and nucleation is proposed to rationalise these findings. The exponential decay of the average solute content predicted by the new model is further analysed and compared with a range of experimental data and contrasted with other models. The new model is found to correspond excellently to these solute decay data.
\end{abstract}

\section{Introduction}

Diffusion-controlled precipitation reactions can be thought of as the combination of 4 consecutive partially overlapping processes: nucleation, growth, soft impingement and coarsening. Substantial data on diffusion-controlled precipitation reactions has been published and several models have been proposed [1]. Very recently [1] a new model 
was derived which focusses particularly on the impingement of diffusion fields. A comparison with of over 20 sets of transformation vs time data at a wide range of temperatures $\left(40-1500^{\circ} \mathrm{C}\right)$ showed that the new model accurately fits the data on all reactions, and overall is clearly outperforming all other models. A key factor in the model is that it derives and employs an expanded form of the imaginary extended volume concept (see e.g. $[2,3,4,5,6]$ ), through applying an integration of impingement over various extended volumes defined by iso-composition surfaces [1]. Through this approach the new model avoids the over-simplification of soft impingement inherent in mean field approaches such as the Kampmann and Wagner (KW) model $[7,8,9,10,11]$ and the model by Svoboda and co-workers $[12,13,14]$.

The main aim of the present paper is to show the consequences of this new model for analysis of nucleation, and to derive methods that can be used to analyse the transformation modes of the diffusion-controlled reactions. An ancillary aim is to show, through these analyses, that the new model is correct, and can form the basis for a range of analysis methods.

For the full derivation of the model the reader is referred to [1]. Whilst the resulting equations are straightforward (see below), a graphical representation of the model is very difficult and the underlying concepts are challenging. To illustrate the 3 dimensional growth of particles one would need to represent each particle growing in its own 3 dimensional space with each point in 3D space having a time dependent composition. This is not realistically possible. Hence we here attempt to represent 1 dimensional growth. Fig. 1a shows the composition profile of one particle shortly after it nucleated. There is one growth dimension: along the $x$ axis. To reflect the assumptions in [1] the composition profile is taken as straight lines. Fig. 1b represents the system in the extended space/volume ${ }^{1}$ a short time later; there are now 2 particles that are growing. As we are formulating the model in the extended space/volume, each particle is associated with its own composition profile in the surrounding matrix that is developing independent of diffusion fields around other particles. Thus in the extended space/volume each particle grows in its own space, which is reflected in Fig. $1 \mathrm{~b}$ and $\mathrm{c}$ by a set of graphs each reflecting one particle.

\footnotetext{
${ }^{1}$ The basic concept of and terminology 'extended volume' derives from the JMAK model $[2,3,4]$. In this paragraph and Fig. 1 the extended 'volume' is illustrated for one dimension. In this paragraph the 'volume' is thus a 1 dimensional 'space'.
} 
As time progresses, the diffusion fields around each particle become wider. Fig. 1c shows the system in the extended volume at a later time; there are now 4 particles that are growing. Fig. $1 \mathrm{~d}$ shows the composition profile in the real space, with composition profiles determined from the transformation from extended to real space (as described in [1]). Whilst the straight line approximation for the composition profiles have introduced an approximation in the model, the resulting composition profile (Fig. 1d) is complex and realistic. It is seen that the model avoids simplifications that have been used in selected other models: particles are randomly distributed (they are not in equidistant arrays) and there is no mean field concentration assumption used in approximating interaction.

In the new model, the fraction transformed, $\alpha$, is calculated on the basis of the average amount of solute in the parent phase, $\bar{c}(t)$, i.e. it is calculated as

$$
\alpha=\frac{c_{o}-\bar{c}(t)}{c_{o}-c_{m}}
$$

where $c_{m}$ is the solute concentration at the precipitate/matrix interface. The results can be described by just 3 basic equations:

$$
\begin{aligned}
& \alpha=\frac{\exp \left(-2 \alpha_{e x t}\right)-1}{2 \alpha_{e x t}}+1 \\
& \alpha_{e x t}=\frac{(k t)^{n}}{V_{o}}
\end{aligned}
$$

where $\alpha$ is the fraction of material that is transformed, $\alpha_{\text {ext }}$ is the extended volume fraction, $k$ is a factor depending on temperature, $t$ is the time, $V_{0}$ is the reference volume considered, and $n$ is an exponent (generally referred to as either the reaction exponent or the 'Avrami exponent'). The general equation for $n$ is $[15,16,17,18]$ :

$$
n=N_{\mathrm{dim}} g+B
$$

where $g$ is $1 / 2$ for diffusion-controlled (parabolic) growth, $B$ is 0 in the case where nucleation ceases very early in the reaction, or 1 for continuous nucleation (at constant nucleation rate in the extended volume), $N_{\text {dim }}$ is the dimensionality of the growth. For diffusion-controlled growth $n$ is thus taken as $1 / 2,1$ or $1 \frac{1}{2}$. 

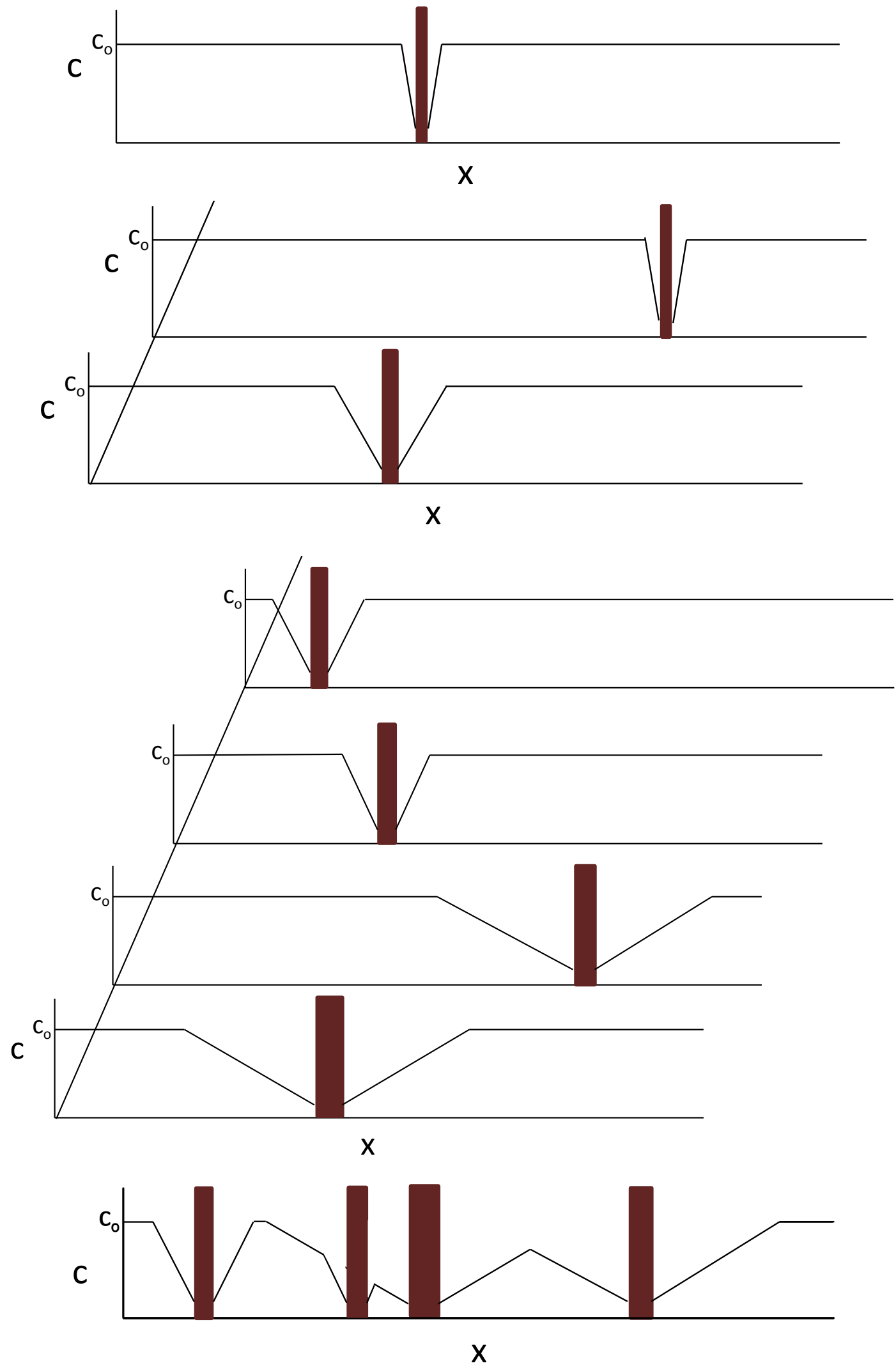

Fig. 1. Schematic illustration of the model for 1 dimensional growth. See text for description. The initial concentration of solute in the alloy is $c_{0}$. 


\section{Methods for direct determination of $n$}

\subsection{Plot slope method}

The main equation in the Johnson-Mehl-Avrami-Kolmogorov (JMAK) model (after $[2,3,19]$, see also $[16,20]), \alpha=1-\exp \left(-\alpha_{\text {ext }}\right)$, can be readily inverted to express $\alpha_{\text {ext }}$ in terms of $\alpha$. This allows calculation of $\alpha_{\text {ext }}$ from data on $\alpha$ and allows plotting $\ln \alpha_{\text {ext }}(\alpha)$ vs $\ln t$. This is the oft employed plot of $\ln t$ vs $\ln (-\ln (1-\alpha))$ (sometimes referred to as an 'Avrami plot'$^{\prime}$ ), which allows determination of $n$ from the slope. (Expressions for selected other models can also be inverted [17,21,22,23]). This 'Avrami plot' method has been applied extensively throughout many works and the $n$ values thus obtained have been extensively discussed in these works. It would appear to be an attractive method, because comparison of $n$ determined from the slope of the plot with Eq. 4 provides information on dimensionality of the growth and nucleation. Even though this is clearly incorrect for diffusion-controlled reactions for all but very small values of $\alpha$, plotting of Int vs $\ln (-\ln (1-\alpha))$ has been applied for these reactions. To rectify this situation, and provide a correct means for determination of $n$ for diffusion-controlled reactions, we will here provide a correct expression for deriving $n$ from slopes of plots for the case of diffusion-controlled reactions.

The new model (Eqs. 2-4) does not allow inverting the main expression (Eq. 2) to provide a simple expression for $\alpha_{\text {ext }}(\alpha)$. However, it is possible to derive highly accurate approximations for this purpose. Through trial and error it was found that a suitable approximation is

$$
\ln \alpha_{\mathrm{ext}}(\alpha) \cong 0.48 \ln \left(\left((1-\alpha)^{1.8}-1\right) /-1.8\right)+0.525 \ln \left(\left((1-\alpha)^{-1.8}-1\right) / 1.8\right)
$$

Analysis of this equation shows that it allows approximation of the local slope of an $\ln \alpha_{\text {ext }}(\alpha)$ vs Int plot (which equals $n$ ) to within $2.3 \%$ for $\alpha$ between 0 and 0.9 (and to within 5\% for $\alpha$ between 0 and 0.97$)^{2}$. The average slope for $\alpha$ between 0.02 to 0.8 equals $n$ to within $0.3 \%$, and the average slope for $\alpha$ between 0.02 to 0.97 equals $n$ to within $0.6 \%$. These levels of variation are generally less than errors and uncertainties introduced by experimental factors, and hence the above approximation can be used to analyse experimental data without adding to experimental errors.

${ }^{2}$ A plot of $\ln t$ vs $\ln (\alpha /[1-\alpha])$ is also interesting as the slope equals $n$ at $\alpha=0$ and $\alpha=1$. But in rises to $1.23 n$ at $\alpha=0.7$ and hence less suitable for determination of $n$. 


\subsection{Time range methods}

Whilst the plot slope method in the previous section is in many cases the most accurate and detailed method available for determining $n$, also faster methods based on transformation time ratios are possible. These methods are possible because, just like in the JMAK method, for each pair of fraction transformed the ratio of the corresponding ratio of times at which these fractions are achieved is a function of only $n$. In formula form this means that for all diffusion-controlled reactions that correspond to Eqs. 2-4:

$$
\ln \left(t\left(\alpha_{2}\right) / t\left(\alpha_{1}\right)\right)=1 / n \ln \left(g\left(\alpha_{2}\right) / g\left(\alpha_{1}\right)\right)
$$

where $g$ is a function that represents the inverse of equation 2. From the latter equation follows:

$$
n=\left(\ln g\left(\alpha_{2}\right)-\ln g\left(\alpha_{1}\right)\right) /\left(\ln t\left(\alpha_{2}\right)-\ln t\left(\alpha_{1}\right)\right)
$$

By numerical solution (using Eqs 2 and 3), we can find these useful relations ${ }^{3}$ for analysing $n$ :

$n=3.842 / \ln (t(\alpha=0.9) / t(\alpha=0.1))$ and $n=2.370 / \ln (t(\alpha=0.8) / t(\alpha=0.2))$.

For experiments in which the rate of transformation is measured (for instance calorimetry) it is valuable to have methods that are based on the time needed to reach the maximum transformation rate, $t_{p}$, and times needed to reach a certain fraction of that peak transformation rate, for instance the time at which the transformation rate has reduced to half the transformation maximum transformation rate. We will denote the latter times as $t_{0.5 \mathrm{p} 1}$ and $t_{0.5 \mathrm{p} 2}$, i.e. for $t_{0.5 \mathrm{p} 1}<t_{\mathrm{p}}, t_{0.5 \mathrm{p} 1}=t\left(\mathrm{~d} \alpha / \mathrm{d} t=0.5(\mathrm{~d} \alpha / \mathrm{d} t)_{\max }\right)$. The concepts are also illustrated in Fig. 2 . The ratios of these characteristic times depend only on $n$ and hence can be used to determine $n$. By numerical solution (using Eqs $2-4$ ), we can find these useful relations:

$$
(n-1)^{0.75} \cong 1.87 / \ln \left(t_{0.5 p 2} / t_{0.5 p 1}\right)
$$

${ }^{3}$ An alternate means to present such relations is to apply Eq. 2 from which we find $n=\frac{\ln \left(h\left(\alpha_{2}\right)\right)-\left(h\left(\alpha_{1}\right)\right)}{\ln \left(t\left(\alpha_{2}\right)\right)-\left(t\left(\alpha_{1}\right)\right)}$ where function $h$ would be determined from Eq. 2. Whilst such an approach is potentially more transparent, the relations presented in the main text are more accurate because they do not rely on an approximations function $h(\alpha)$. 
The ratio $t_{0.5 \mathrm{p} 2} / t_{0.5 \mathrm{p} 1}$ can be termed the times at half maximum ratio (THMR): it is effectively a measure of the width of curve in the $\mathrm{d} \alpha / \mathrm{d} t$ vs $\ln t$ plot. For reactions in which the start of the reaction is difficult to discern (possibly due to overlap with a preceding reaction) it is useful to be able to analyse $n$ based on $t_{0.5 \mathrm{p} 2} / t_{\mathrm{p}}$. For this ratio, numerical solution (using Eqs 2 - 4) yields:

$$
(n-1)^{0.64} \cong 0.71 / \ln \left(t_{0.5 p 2} / t_{p}\right)
$$

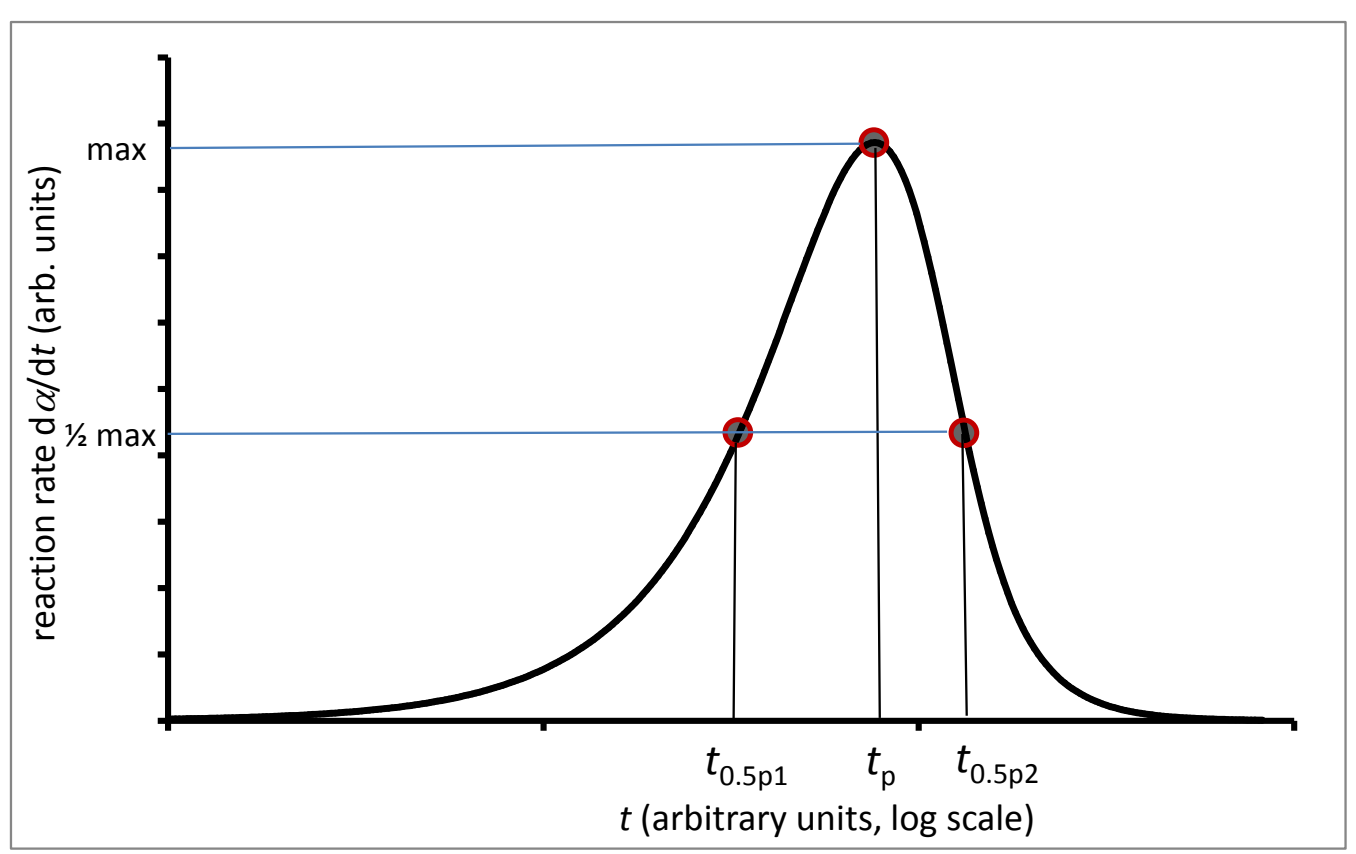

Fig. 2. Graph of the reaction rate, $\mathrm{d} \alpha / \mathrm{d} t$, vs $t$, for the new model, illustrating the characteristic times $t_{p}, t_{0.5 p 1}$ and $t_{0.5 p 2}$.

\section{Determination of $\boldsymbol{n}$ for a range of diffusion-controlled reactions.}

To test the above plot slope method for determination of $n$, data on a wide range of diffusion-controlled reactions, ranging from Al-based alloys, to steels and $\mathrm{Ni}$-based alloys were collected. The sources of the data are [24,25,26,27,28,29,30,31,32,33]. Further details of this data collection process are provided in [1]. For all reactions the precipitates formed throughout grains and no particular propensity for nucleation on defects was reported.

In Fig. 3 plots of $0.48 \ln \left(\left[(1-\alpha)^{1.8}-1\right] /-1.8\right)+0.525 \ln \left(\left[(1-\alpha)^{-1.8}-1\right] / 1.8\right)$ vs $\ln t$ are provided for a number of diffusion-controlled reactions reported in $[24,25,26,27,28]$. Only 
reactions with 3D diffusion are considered and reactions with $n$ close to 2.5 (as determined in [1]) are not considered in Fig 1. As predicted by the new model, the plots in Fig. 3 are straight lines. (With some limited deviations and scatter due to experimental errors noticeable for small $\alpha$ (mostly $\alpha<0.04$ ) and on approach of completion of reaction (particularly $\alpha>0.96$ ).) Evaluation of the slopes between $\alpha=0.04$ and 0.96 reveals that the data sets have slopes of $1.48 \pm 0.06$, i.e. they correspond excellently with the model which requires $n=1 \frac{1}{2}$ for these reactions.

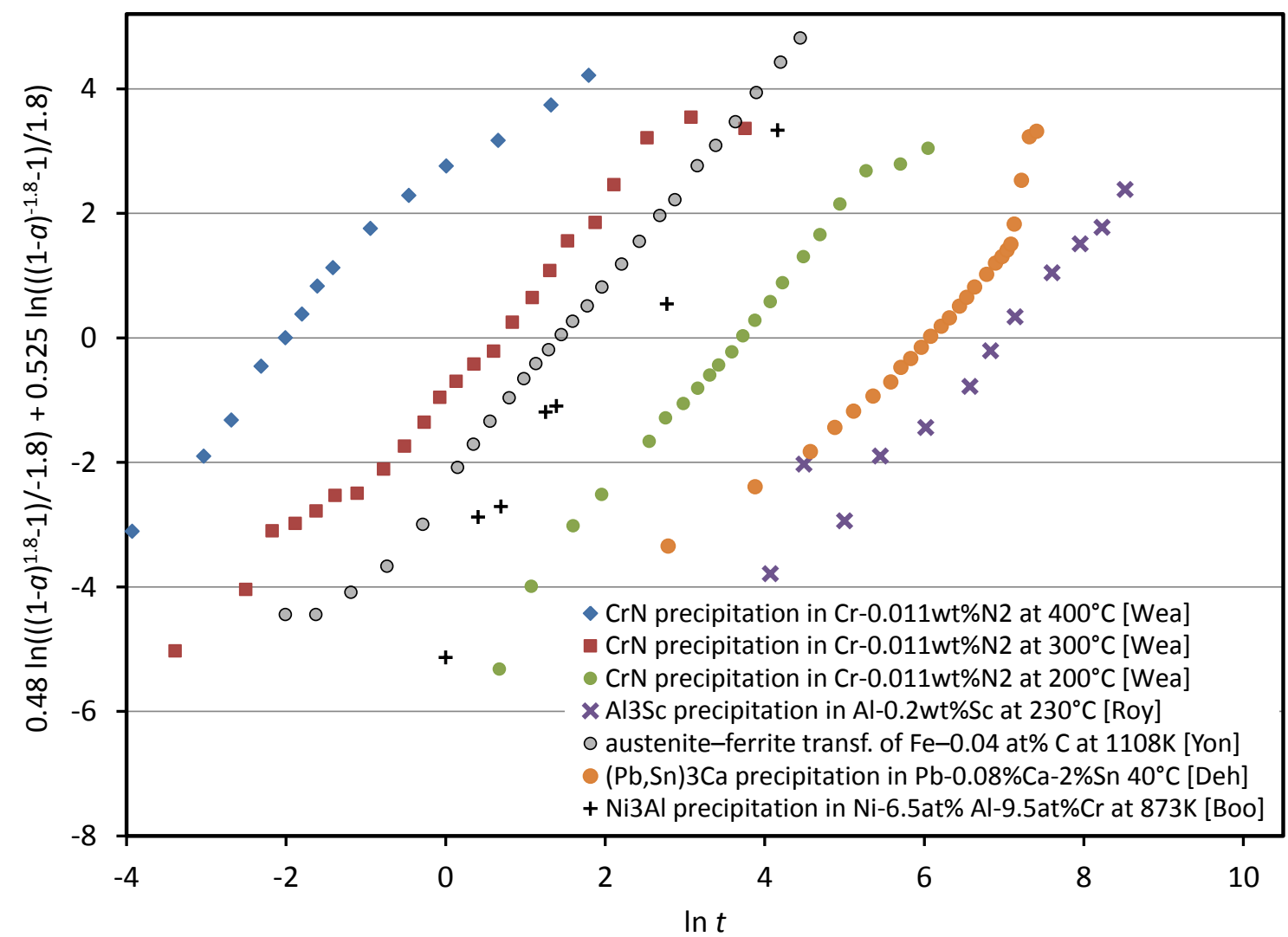

Fig. 3. Plot of approximation of $\ln \alpha_{\text {ext }}\left(\cong 0.48 \ln \left(\left((1-\alpha)^{1.8}-1\right) /-1.8\right)+0.525 \ln \left(\left((1-\alpha)^{-1.8}-1\right) / 1.8\right)\right)$ vs Int for a range of diffusion-controlled reactions. Note that plots are in good approximation straight between $\alpha=0.04\left(\ln \alpha_{\mathrm{ext}}=-3.2\right)$ and about $\alpha=0.96\left(\ln \alpha_{\mathrm{ext}}=2.5\right)$. Data from [Wea] $=[24]$, $[$ Roy] $=[25],[$ Yon] $]=[26],[$ Deh] $=[27],[$ Boo] $]=[28]$.

A range of further data was analysed using the time range methods (Eqs. 6 to 9). The data is collected in Table 1. (For reactions in Al-6Si alloys the initial part of the main precipitation reaction shows an overlap with a prior reaction [33] and hence the main reaction was analysed using the $t_{0.5 \mathrm{p} 2} / t_{\mathrm{p}}$ method, which avoids the initial stage of the reaction.) 
Table 1 Reactions analysed and $n$ values determined using the methods outlined in Section 3.

\begin{tabular}{|c|c|c|c|c|c|c|}
\hline Alloy & $\begin{array}{l}\text { Phase } \\
\text { formed }\end{array}$ & $\begin{array}{l}\text { Source } \\
\text { of data }\end{array}$ & method & $T\left({ }^{\circ} \mathrm{C}\right)$ & $T_{\text {st }}\left({ }^{\circ} \mathrm{C}\right)$ & $n$ \\
\hline Cr-(0.01-0.014)\%N & $\mathrm{CrN}$ nitride & [24] & plot slope method & 200 & 950 & 1.46 \\
\hline Cr-(0.01-0.014)\%N & $\mathrm{CrN}$ nitride & [24] & plot slope method & 300 & 950 & 1.37 \\
\hline $\mathrm{Pb}-0.08 \% \mathrm{Ca}-2 \% \mathrm{Sn}$ & $(\mathrm{Pb}, \mathrm{Sn})_{3} \mathrm{Ca}$ & [27] & plot slope method & 40 & 327 & 1.42 \\
\hline Cr-(0.01-0.014)\%N & $\mathrm{CrN}$ nitride & [24] & plot slope method & 400 & 950 & 1.57 \\
\hline $\mathrm{Ni}-6.5$ at\% Al-9.5 at\% Cr & $\mathrm{Ni}_{3} \mathrm{Al}$ & [28] & plot slope method & 600 & 770 & 1.49 \\
\hline Al-6Si & $\mathrm{Si}$ & [33] & $t_{0.5 \mathrm{p} 2} / t_{\mathrm{p}}$ method & 190 & 550 & 1.52 \\
\hline Al-6Si & $\mathrm{Si}$ & [33] & $t_{0.5 \mathrm{p} 2} / t_{\mathrm{p}}$ method & 210 & 550 & 1.39 \\
\hline Al-6Si & $\mathrm{Si}$ & [33] & $t_{0.5 \mathrm{p} 2} / t_{\mathrm{p}}$ method & 230 & 550 & 1.40 \\
\hline $\mathrm{Al}-0.2 \% \mathrm{Sc}$ & $\mathrm{Al}_{3} \mathrm{Sc}$ & [25] & plot slope method & 230 & 365 & 1.50 \\
\hline $\mathrm{Al}-0.2 \% \mathrm{Sc}$ & $\mathrm{Al}_{3} \mathrm{Sc}$ & [25] & plot slope method & 270 & 365 & 2.25 \\
\hline Al-5.8at\%Zn & GP zone & [1] & $t_{0.8} / t_{0.2}$ method & 130 & 200 & 2.5 \\
\hline Ti0.3W0.4Cr0.3B2 & $\mathrm{W}_{2} \mathrm{~B}_{5}$ & [31] & plot slope method & 1500 & 1750 & 2.55 \\
\hline Al- $0.2 \%$ Sc & $\mathrm{Al}_{3} \mathrm{Sc}$ & [25] & plot slope method & 310 & 365 & 2.4 \\
\hline Al-0.2wt.\% Sc & $\mathrm{Al}_{3} \mathrm{Sc}$ & [25] & plot slope method & 330 & 375 & 2.5 \\
\hline Fe-0.04at\%C & ferrite & [26] & plot slope method & 835 & 912 & 1.5 \\
\hline Al-1.8Cu-1.5Mg-0.2Mn & co clusters & {$[30,31]$} & plot slope method & 25 & 380 & 2.48 \\
\hline Al-1.2Cu-1.2Mg-0.2Mn & co clusters & {$[30,31]$} & plot slope method & 25 & 320 & 2.4 \\
\hline Al-0.9Cu-1.5Mg-0.03Zr & co clusters & [30] & plot slope method & 25 & 300 & 2.5 \\
\hline
\end{tabular}

\section{$4 \quad$ Discussion and analysis}

\subsection{Analysis and verification of treatment of impingement.}

In order for the methods outlined in Section 3 to be reliable, we need to be sure that the new kinetic model for diffusion-controlled reactions is correct and specifically we need to be sure that the treatment of soft impingement is reliable. The extensive model verification through comparison with over 20 sets of transformation amount vs time data reported in [1] provided a first key test. In the present work, the straight lines in Fig. 3 all with slope close to $1 \frac{1}{2}$ is a further indication the model is sound, and also the observation that all values of $n$ for diffusion-controlled reactions with product phases 
growing in 3 dimensions determined are close to $1 \frac{1}{2}$ or $2 \frac{1}{2}$ (Table 1 ) supports the model. The data in Table 1 will be further discussed in Section 4.2, but in this section we will first compare in further detail the experimental data on the impingement stage with the model predictions.

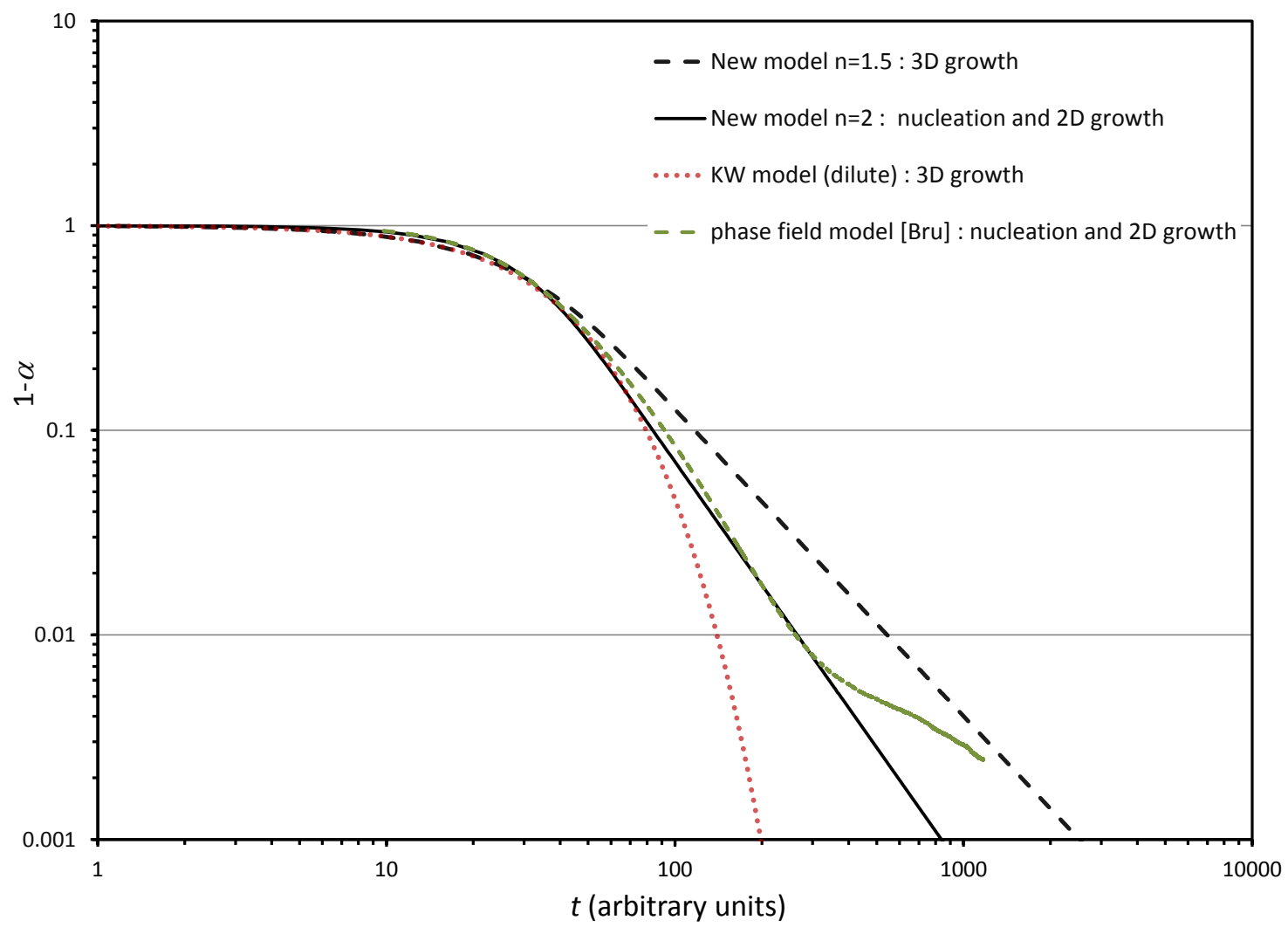

Fig. 4. Plot of (1- $\alpha)$ vs $t$ for a range models. The new model shows a linear asymptotic behaviour on a log-log scale which is consistent with 2D phase field model predictions by Bruna et al [34] (up to $\alpha=0.995$ ). The mean field model (KW model) does not show this asymptotic behaviour.

The new model predicts that for the impingement stage $(\alpha>0.3),(1-\alpha)$ will show a linear time decay on a log-log plot [1]. In Fig. 4 such a log-log plot is presented for the model with $n=1 \frac{1}{2}$ and $n=2$ and it is compared with a 2D phase field model of a diffusioncontrolled reaction with continuous nucleation (data from Bruna et al. [34]). The new model with $n=2$ should coincide with that (computationally expensive) phase field model, and indeed the correlation is good from $\alpha=0$ up to $\alpha=0.995$. This provides further confidence in the present new model. The predictions by the KW [7] model (for constant number of nuclei, i.e. without dissolution of particles in a coarsening stage) are also presented in Fig. 4. Whilst initially (up to about $\alpha=0.3$ ) this model coincides with the present new model for $n=1 \frac{1}{2}$, it does not show a linear decay on a log-log plot, and 
deviations between the models become very large. It has been suggested that the mean field approximation underestimates soft impingement $[1,35]$.

In Fig. 5, a log-log plot of $(1-\alpha)$ vs $t$ is presented for a range of diffusion-controlled reactions (data from $[24,25,26,27,28,36]$ ), and for comparison predictions from the new model (for $n=1^{\frac{1}{2}}$ and $n=2^{\frac{1}{2}}$ ) as well as the KW model (for constant number of nuclei, i.e. without dissolution of particles in a coarsening stage) are presented. Considering the typical accuracy in determination of $\alpha$ is about 0.01 for the data considered here, Fig. 5 shows the experimental data is in very good agreement with the new model for $n=1 \frac{1}{2}$. The plot also illustrates that that the KW model does not fit to the experimental data.

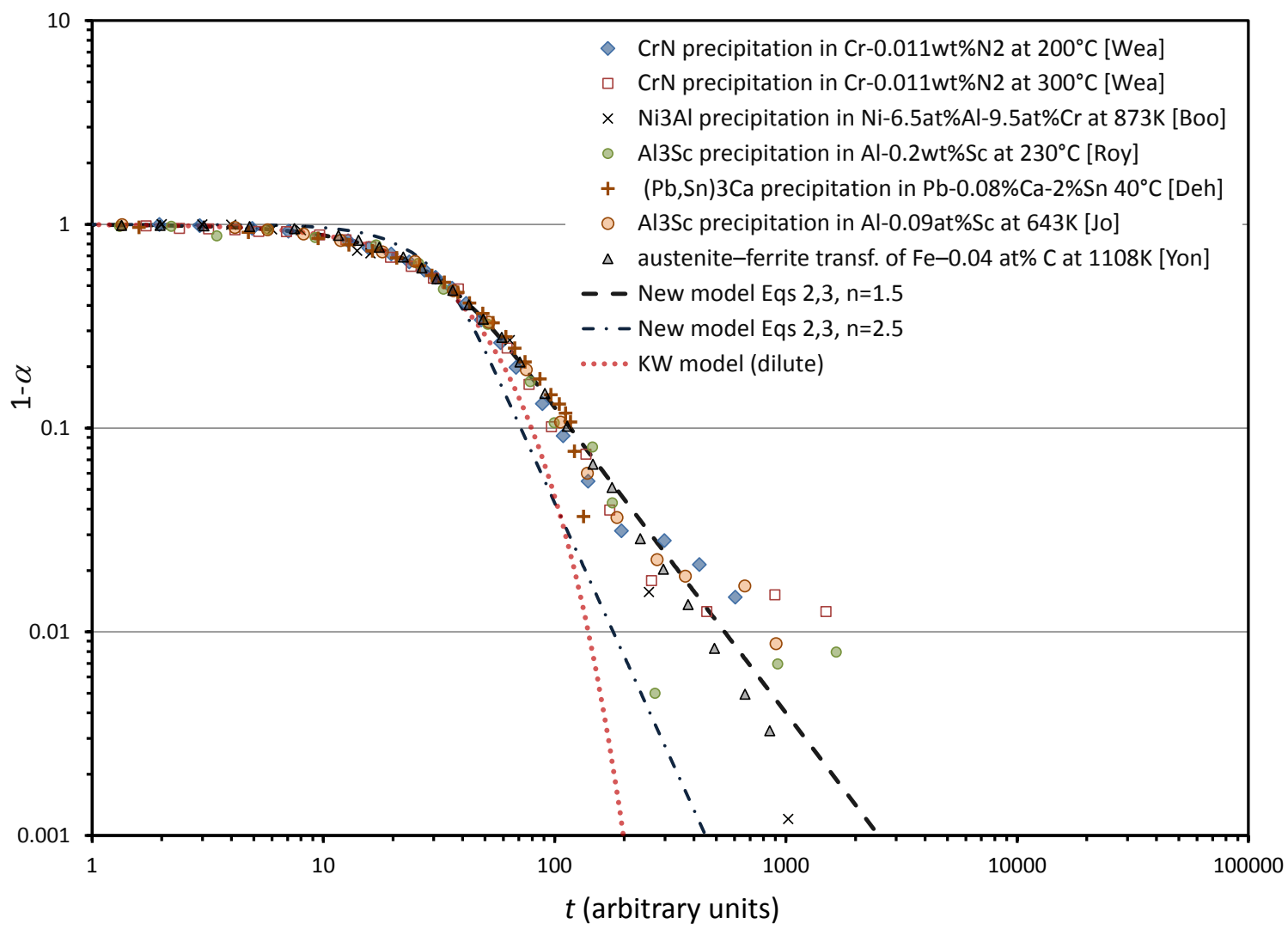

Fig. 5. Plot of (1- $\alpha)$ vs $t$ for a range of diffusion-controlled reactions. Time is rescaled such that $t(\alpha=0.5)$ coincides. Data from [Wea]=[24], [Roy]=[25], [Yon]=[26], [Deh]=[27] , [Boo]=[28], [Jo]=[36]. (Note that experimental error in $\alpha$ is typically about 0.01 , i.e. on this log scale error bars would increase in size towards the bottom of the plot).

\subsection{The relation between reaction exponent $n$, nucleation, undercooling and phonons.}

With the analysis methods based on the new model a reliable determination of the reaction exponent $n$ in diffusion-controlled reactions is now possible, and the obtained 
$n$ values can form the basis of a sound analysis. A survey of the values presented in Table 1 shows that $n$ is nearly always very close to $1 \frac{1}{2}$ or $2^{\frac{1}{2}}$. In classical nucleation theory, nucleation rates are considered to be closely related to undercooling, and hence it was decided to plot the determined values of $n$ as a function of the ratio of transformation (reaction) temperature, $T_{\mathrm{tr}}$, and the temperature of maximum stability of the reaction product phase (i.e. the precipitate phase) in the alloy, $T_{\mathrm{s}} . T_{\mathrm{s}}$ was estimated from solvus data of the Ni-Al system [37], data of the Pb-Ca system [38], TTT plots of precipitation in Al-Sc [25], the thermodynamic model for $\mathrm{Cu}-\mathrm{Mg}$ co-clusters in Al-Cu-Mg alloys $[39,40]$, the Fe-C system phase diagram, the Al-Si phase diagram and the estimated solvus of $\mathrm{CrN}$ by Weaver [24]. Data on $T_{\mathrm{s}}$ thus obtained is included in Table 1.

The plot of $n$ vs $T_{\mathrm{tr}} / T_{\mathrm{s}}$ (Fig. 6) shows a clear trend: for $T_{\mathrm{tr}} / T_{\mathrm{s}}<0.84$ the reactions involving long range ordered phases proceed with $n=1.5$, i.e. nucleation is confined to the very early stages and no significant nucleation occurs during the vast majority of the transformation, whilst for high $T_{\mathrm{tr}} / T_{\mathrm{s}}\left(T_{\mathrm{tr}} / T_{\mathrm{s}}>0.84\right)$ the reaction proceeds with $n=2.5$, i.e. nucleation occurs continuously during the entire reaction. For co-cluster formation (short-range ordered structures) all $n$ values are found to be close to 2.5. Fig. 6 suggests that there is a sharp transition between the two transformation modes ( $n=1^{\frac{1}{2}}$ or $2^{\frac{1}{2}}$ ) for long-range ordered precipitates.

The present findings indicate that diffusion-controlled reactions involving nucleation within a grain and growth in 3 dimensions have only two distinct modes that are stable: $n=1 \frac{1}{2}$ and $n=2 \frac{1}{2}$. These two modes reflect nucleation that is concentrated in the very early stages of the reaction and continuous nucleation in which nucleation occurs at a constant rate in the extended volume. Indeed, available experimental data on precipitate density in a range of systems indicates that the density of precipitates is close to constant during many transformations for nearly all of the transformation (see [41] for ageing of a Nb-V micro-alloyed steel, Booth-Morrison et al. [28] for ageing of $\mathrm{Ni}-6.5$ at\%Al-9.5 at\% $\mathrm{Cr}$ at $873 \mathrm{~K}$, [42] for precipitation in an Fe-Si-Ti alloy and Schober et al. [43] for precipitation of intermetallic NiAl particles in Fe-6 at\%Al-4 at\%Ni). The existence of only two modes ${ }^{4}$ suggests that the classical models for nucleation, which

\footnotetext{
${ }^{4}$ In some older literature the term 'site saturated' nucleation is used to describe processes with $B=0$, for instance 3 dimensional growth with $n=1 \frac{1}{2}$. This 'site saturation' can occur for heterogeneous nucleation where the number of nucleation sites is limited, but this seems to not
} 
generally allow a continuous variation in nucleation rate, cannot fully capture the mechanism. Whilst a full discussion of nucleation models is beyond the scope of the present paper, there are few points that need to be considered with regards to the relation between the present findings and classical nucleation theories.

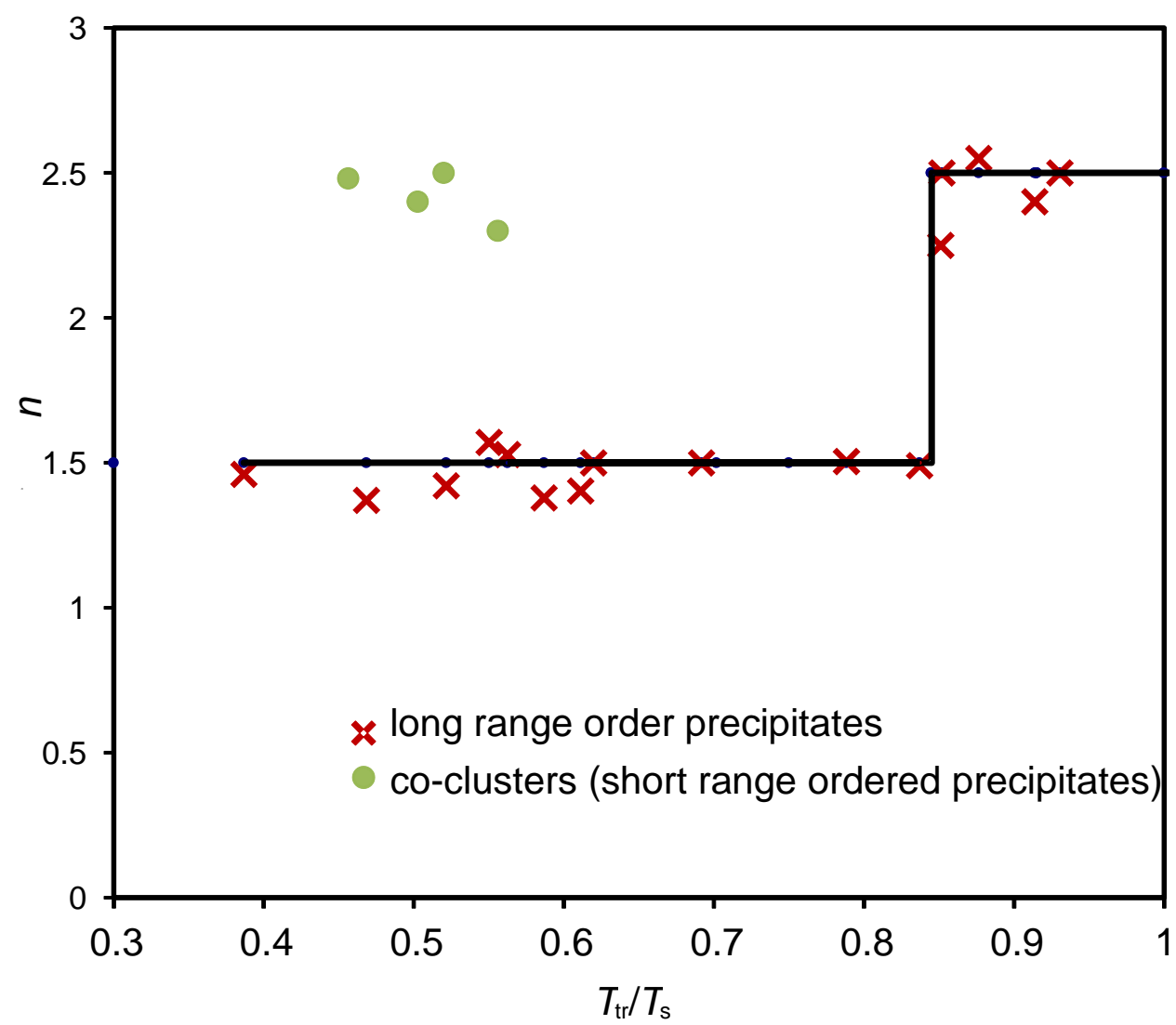

Fig. 6. Reaction exponent $n$ for reactions in Table 1 as a function of the ratio of the transformation temperature $T_{\mathrm{tr}}$ and is the maximum temperature of stability $T_{\mathrm{s}}$.

In classical nucleation models, the probability of nucleation at a particular location is determined by parameters such as local solute concentration, (average) thermodynamic temperature, a (local) diffusion rate, a local energy barrier and an interfacial energy. As a result, a diffusion event causing growth of a precipitate in a nearby location does not influence the nucleation probability other than that it will over time reduce the solute content and hence over time reduce the nucleation rate. In such a model the

be the case for the reactions studied here: there is no indication in microstructural studies on these alloys that there is a limit on the number of sites. 
interaction determining the rates of the two processes (nucleation and growth) occurs through diffusion of atoms, which has a speed that is limited to the diffusion rate. Thus in such a model nucleation and growth are concurrent processes which are linked only through diffusion and both nucleation and growth change gradually in the course of the transformation. The present findings appear to challenge this classical view: the data in Table 1 and Fig. 3 indicate that nucleation it is either 'on' (at a constant rate in the extended volume) or 'off', i.e. after an initial burst of nucleation to start the reaction, the nucleation rate becomes negligibly small during nearly the entire transformation. We can rationalise this finding by considering that nucleation at each location is closely linked to growth of nearby precipitates, and the system will evolve by either using all free energy available for overcoming energy barriers to grow existing particles (i.e. no or negligible nucleation) or provide the maximum nucleation rate possible. To enable this to happen there needs to be a mechanism through which energy is exchanged at a high speed between potential nucleation sites (i.e. the entire parent phase) and growing particles. Such mechanisms exist: phonons (lattice vibrations) travel at the speed of sound and also diffusion of vacancies is much faster than diffusion of atoms. Thus the system as a whole can switch between a maximum nucleation rate process and zero nucleation (growth only), depending on which balance produces the fastest decrease in free energy of the system. This explains the finding of the existence of only 2 modes in Fig. 6.

The reaction for the formation of co-clusters in Al-Cu-Mg alloys forms an exception to the trends seen in Fig. 6: for this reaction $n$ appears to be constant at 2.5 independent of $T_{\mathrm{tr}} / T_{\mathrm{s}}$. Also this is consistent with the above interpretation as for these short range ordered structures the change in free energy is determined by the $\mathrm{Cu}-\mathrm{Mg}$ bonds formed $[39,40,44,45]$ and hence only continued formation (nucleation) of Cu-Mg bonds can reduce the free energy of the system.

It is also interesting to closer examine the dataset for the reaction appearing closest to the point where the mode switches at $T_{\mathrm{tr}} / T_{\mathrm{s}}=0.84$. For the formation of $\mathrm{L}_{2}$ ordered $\mathrm{Ni}_{3} \mathrm{Al}$ precipitates in the $\mathrm{Ni}-6.5$ at\%Al-9.5at\% $\mathrm{Cr}$ alloy at $873 \mathrm{~K}$ (from [28]), which is the reaction with the highest $T_{\mathrm{tr}} / T_{\mathrm{s}}$ amongst the reactions with $n=1.5$, there is evidence both in Fig. 3 and in the original paper [28] that a brief transient rapid nucleation occurs for $\alpha$ up to 0.03 . Fig. 3 shows that it is the only dataset in which a high slope (i.e. high $n$ ) is 
seen before the dominant $n=1.5$ slope sets in for the majority of the reaction, whilst in the original paper [28] precipitate density measurement using atom probe tomography (a.k.a. 3 dimensional atom probe) also shows this brief transient rapid nucleation. These observations are consistent with the present analysis: for this borderline case rapid nucleation can be detected for a small range of the reaction, before the predominant mode (growth at constant number of precipitates) takes hold.

\section{Conclusions}

A new model for diffusion-controlled reactions was further analysed and methods for deriving the reaction mode in terms of the reaction exponent $n$ were derived. Conclusions can be drawn as follows:

- Analysis of the exponential decay of (1- $\alpha)$ for a range of reactions provides further proof that the new model is accurate, and conclusively shows that models based on mean field approaches do not fit to this extensive data.

- Analysis of the model for diffusion-controlled reactions shows that these reactions can be analysed by plotting $0.48 \ln \left(\left((1-\alpha)^{1.8}-1\right) /-1.8\right)+0.525 \ln \left(\left((1-\alpha)^{-1.8}-1\right) / 1.8\right)$ vs $\ln \mathrm{t}$.

The slope provides the reaction exponent $n$, which equals $n=N_{\mathrm{dim}} g+B$, where $g$ is $\frac{1}{2}$ for parabolic (diffusion-controlled) growth, $B$ is 0 in the case where nucleation ceases very early in the reaction, or 1 for continuous nucleation (at constant nucleation rate), $N_{\text {dim }}$ is the dimensionality of the growth.

- $n$ can also be analysed using time range methods such as $n=3.842 / \ln (t(\alpha=0.9) / t(\alpha=0.1))$ and $n=2.370 / \ln (t(\alpha=0.8) / t(\alpha=0.2))$

- Consistent with the $n=N_{\text {dim }} g+B$ interpretation, determination of $n$ for a large number of reactions shows that $n$ is always $1 \frac{1}{2}$ or $2^{\frac{1}{2}}$. This provides a consistent analysis where reaction model and $n$ value are linked in a consistent way.

\section{Acknowledgements}

Dr. D. Crespo is gratefully acknowledged for providing the phase field model data displayed in Fig 4.

\section{References}

1 M.J. Starink, Thermochim Acta 596, 2014, 109-119. 
2 M. Avrami. J Chem Phys 8, 1940, 212.

3 A.N. Kolmogorov. Izv Akad Nauk SSSR Ser Mater 1, 1937; 355.

4 A.A. Burbelko, E. Fraś, W. Kapturkiewicz. Mater Sci Eng A 413-414, 2005; 429-434.

5 M.J. Starink. J Mater Sci 32, 1997; 4061.

6 M. Tomellini, Thermochim Acta 566, 2013, 249-256.

7 R. Kampmann, R. Wagner. in Decomposition of Alloys: the Early Stages, Haasen P, Gerold V, Wagner R, Ashby MF (Eds.), New York, Pergamon Press, 1984, 91-103.

8 O.R. Myhr, $\varnothing$ Grong. Acta Mater 48, 2000, 1605.

9 J.D. Robson. Acta Mater 52, 2004; 1409-1421.

10 I.N. Khan, M.J. Starink, J.L. Yan. Mater Sci Eng A 472, 2008, 66-74

11 Qiang Du, Yanjun Li, Acta Mater, 71, 2014, 380-389.

12 J. Svoboda, F.D. Fischer, P. Fratzl, A. Kroupa, Acta Mater. 50, 2002, 1369.

13 J. Svoboda, E. Gamsjàger, F.D. Fischer, P. Fratzl. Acta Mater 52, 2004, 959-967

14 E. Kozeschnik, J. Svoboda, P. Fratzl , F.D. Fischer, Mater Sci Eng A, 385, 2004, 157 - 165.

15 M.J. Starink. J Mater Sci, 36, 2001, 4433-4441.

16 F.L. Cumbrera, F. Sánchez-Bajo, Thermochim Acta 1995, 266, 315-330.

17 M.J. Starink. Int Mater Rev, 49, 2004, 191-226.

18 T.A. Baker, O.L.A. Monti, D.J. Nesbitt, J Phys Chem C 115, 2011, 9861-9870.

19 W.A. Johnson, K.E. Mehl, Trans Am Inst Min Met Engrs 195, 1939, 416

20 J.W. Christian. The Theory of Transformation in Metals and Alloys, 2nd ed., Part 1 , Oxford, UK, Pergamon Press, 1975

21 M.J. Starink and A.-M. Zahra, Acta Mater, 46, 1998, 3381-3397.

22 Eon-Sik Lee and Young G. Kim, Acta Metall. Mater., 38, 1990, 1669.

23 M.J. Starink, P. Wang, I. Sinclair, P.J. Gregson, Acta Mater, 47, 1999, 3841-3853

24 C.W. Weaver. Acta Metall 10, 1962, 1151-1160.

25 J Røyset, N Ryum. Mater Sci Eng A 2005; 396, 409-422.

26 Y Liu, D Wang, F Sommer, EJ Mittemeijer. Acta Mater 2008; 56, 3833-3842.

27 M. Dehmas, A. Maitre, J.B. Richir, P. Archambault. J Power Sources 159, 2006, 721-727.

28 C. Booth-Morrison, Y. Zhou, R.D. Noebe, D.N. Seidman. Philosoph Mag 90, 2010, 219-235.

29 M.J. Starink and A.-M. Zahra, Thermochim Acta 298, 1997, 179-189.

30 J. Yan, PhD thesis, Materials Research Group, University of Southampton, UK, 2006.

31 M.J. Starink, N. Gao, L. Davin, J. Yan and A. Cerezo, Philosoph Mag, 85, 2005, 1395-1417.

32 S. Bartels, W. Gruber, B. Cappi, R. Telle, H. Schmidt. Mater Lett 62, 2008, 3836-3838.

33 M.J. Starink and A.-M. Zahra, Philosoph Mag A, 77, 1998, 187-199.

34 P. Bruna, E. Pineda, J.I. Rojas, D. Crespo, J All Comp 483, 2009, 645.

35 P. Bruna, D Crespo, R González-Cinca, E Pineda, J. Appl. Phys. 100 (2006) 054907.

36 Hyung-Ho Jo, S.-I. Fujikawa, Mater Sci Eng A, 171, 1993, 151-161.

37 H. Okamoto, J. Phase Equilibria Diff 25 (2004) 394.

38 G. Bruzzone, F. Merlo, J. Less Common Metals, 48, 1976, 103-109.

39 M.J. Starink, S.C. Wang, Acta Mater, 57, 2009, 2376-2389

40 M.J. Starink, Intern J Mater Research, 8, 2012, 942-947

41 Q. D. Liu, W. Q. Liu, S. J. Zhao. Metals Mater Intern, 18, 2012, 1009-1013

42 M. Perrier, A. Deschamps, O. Bouaziz, Y. Brechet, F. Danoix, F. Geuser, P. Donnadieu, K Hoummada, P. Maugis, Metall Mater Trans A, 43, 2012, 4999-5008

43 M. Schober, Ch. Lerchbacher, E. Eidenberger, P. Staron, H. Clemens, H. Leitner, Intermetall, 18, 2010, 1553-1559.

44 M.J. Starink, L.F. Cao, and P.A. Rometsch, Acta Mater, 60, (2012), 4194-4207.

45 Ying Chen, Nong Gao, Gang Sha, S.P. Ringer, M.J. Starink, Mater Sci Eng A, 627, 2015, 10-20; doi:10.1016/j.msea.2014.12.107 